Original Artikel

\title{
Elektrophoretische Untersuchungen der wasserlöslichen Proteine bei der Honigbiene Apis mellifera L. im Verlauf der Ontogenese
}

\author{
Evgenia Ivanova*, Petar Popov, Ivan Dobrovolov \\ Paissij-Hilendarski-Universität Plovdiv, Biologische Fakultät, \\ Sektion Genetik. Zar Assenstr. 24, Plovdiv, Bulgarien
}

(Received 30 October 1999; revised 15 March 2000; accepted 20 July 2000)

\begin{abstract}
Zusammenfassung - Durch Elektrophorese in Polyacrylamid- und Stärkegel von wasserlöslichen Proteine mit Totalextrakten von Honigbienen konnte eine Veränderung im Vorkommen der Proteine während der Ontogenese der Bienen nachgewiesen werden: Bei PAGE wurden 17 Fraktionen bei Rundmaden, 19 bei Streckmaden und 14 bei Puppen gefunden. Bei den Imagines konnten je 1 spezielle Fraktion für Königinnen und 1 für Drohnen nachgewiesen werden. Im Stärkegel wurden bei Rundmaden 8, bei Streckmaden 11, bei Puppen 10 Fraktionen und jeweils 2 königinnen - bzw. 2 drohnenspezifische Fraktionen nachgewiesen. Durch isoelektrische Fokussierung in dünnem und ultradünnem Polyacrylamidgel gelang eine Feinauftrennung mit bis zu 52 Fraktionen, deren Verteilung ebenfalls variierte.
\end{abstract}

Apis mellifera L. / Ontogenese / wasserlösliche Proteine

\section{EINLEITUNG}

In der Literatur finden sich quantitative Untersuchungen über das Gesamteiwei $\beta$ der Haemolymphe [1, 2, 5, 9, 14, 19] Mitteldarm [23] und der Hypopharynxdrüsen [10, 11, 15, 20, 21] der Bienen. Qualitative Untersuchungen betreffen hauptsächlich das Vorkommen von globulin- und albuminähnlichen Stoffen in der Haemolymphe
$[8,23]$ und insbesondere von Vitellogenin und juvenilen Hämolymphproteinen $[6,16$, 25, 29-31]. Außerdem wurde eine Veränderung der Eiweißzusammensetzung in Abhängigkeit vom verschiedenen Grad der Invasion durch Varroa jacobsonii bei Honigbienen nachgewiesen [4, 7, 13, 26], Unterschiede in Konzentration und Vorkommen der wasserlöslichen Proteine bei Imagines von Apis mellifera wurden in

* Correspondence and reprints 
Abhängigkeit von Geschlecht und Fertilität durch Elektrophorese in Polyacrylamidgel von Popov \& Ivanova [22] und Ivanova [12] festgestellt.

In der vorliegenden Arbeit wird durch verschiedene elektrophoretische Methoden das Auftreten der wasserlöslichen Proteine bei Apis mellifera im Verlauf ihrer Ontogenese untersucht.

\section{MATERIAL UND METHODEN}

Wir untersuchten elektrophoretisch etwa 2000 Individuen verschiedenen Geschlechts von Apis mellifera in verschiedenen Stadien ihrer Individualentwicklung. Wir verglichen Eier, 5 Tage alte Rund- und 7 Tage alte Streckmaden, 3 Tage alte weissäugige und 7 Tage alte dunkeläugige Puppen. Bei Adulten untersuchten wir 7 Tage alte Arbeiterinnen, 10 Tage alte Drohnen und 1-jährige Königinnen. Alle Proben bestanden aus Totalextrakten von Einzeltieren, mit Ausnahme der Proben aus Eiern, bei der 20 Eier pro Versuch genommen wurden. Als Extraktionsmittel wurde $0,1 \mathrm{M}$ Tris-Phosphatpuffer mit pH 6,7 benutzt. Die untersuchte Eiweissmenge in einer Probe war $8,5 \mu \mathrm{g} / \mathrm{ml}$ nach der Methode von Lowry [17]. Wir zentrifugierten bei $900 \mathrm{~g}$ über 45 Minuten für Polyacrylamid- und 30 Minuten für Stärkegel.

Die elektrophoretische Trennung in 7,5\% PAAG führten wir nach Maurer [18] mit einigen Modifikationen durch. Die benutzte Gelplatte hatte eine Kapazität von 23 Proben. Das konzentrierende Gel hatte eine Konzentration von 3,3\% und $\mathrm{pH} 6,7$, das Trenngel 7,5 \% und pH 8,9. Anoden- und Kathodenpuffer der Kammern war 0,05M Tris- 0,2M Glycin mit pH 8,3. Gefärbt wurde mit Coomassie in $14 \%$ iger Trichloressigsäure, fixiert in $7 \%$ iger Essigsäure.

Die Elektrophorese in Stärkegel (SG) wurde nach Smithies [28] in der Modifikation von Dobrovolov [3] durchgeführt. Wir arbeiteten mit $13 \%$ SG, die Hauptpuffer- lösung bei der elektrophoretischen Analyse war $0,05 \mathrm{M}$ Tris-EDTA-Boratpuffer mit pH 8,1. Wir benutzten das unterbrochene Puffersystem nach Dobrovolov [3] mit 0,05M Tris-EDTA-Boratpuffer mit $\mathrm{pH} 8,1$ und $0,3 \mathrm{M}$ Natriumborat-Elektrodenpuffer mit $\mathrm{pH} 8,9$ und $0,155 \mathrm{M}$ Tris-Zitratpuffersystem mit $\mathrm{pH} 8,0$. Wir entwickelten mit Amidoschwarz und bewahrten die Platten in $5 \%$ iger Essigsäure auf.

Die isoelektrische Fokussierung wurde in ultradünnem PAAG durchgeführt. Die Gewebefragmente wurden durch 15 Minuten zentrifugieren bei $900 \mathrm{~g}$ abgetrennt.

Zur Trennung in ultradünnem Gel benutzten wir Firmenplatten Servalyt Precotes mit $\mathrm{pH}$-Gradient von 3 bis 10 und $0,3 \mathrm{~mm}$ Geldicke. Gearbeitet wurde bei $2000 \mathrm{~V}, 10 \mathrm{~mA}$, $6 \mathrm{~W}$ in 3 Stunden.

Die Färbung wurde mit Coomassie Brilliant Blue $\mathrm{R}-250$ bei $60^{\circ} \mathrm{C}$ in $15-20 \mathrm{Min}$. durchgeführt.

\section{ERGEBNISSE}

\subsection{Polyacrylamidgelelektrophorese (PAGE)}

Bei der in PAGE durchgeführte Elektrophorese traten insgesamt 24 Fraktionen wasserlöslicher Eiweisse auf. Dabei erwies sich eine Dynamik ihres Auftretens in Abhängigkeit von Ontogenesestadium, Geschlecht und Fertilität der Individuen als charakteristisch: bei den Sammelproben von Eiern ließen sich 10 Fraktionen, bei Rundmaden 17 Fraktionen, bei Streckmaden 18 Fraktionen, bei Puppen (weiss- und dunkeläugigen) 14 Fraktionen nachweisen. Bei Imagines fanden wir 18 Fraktionen bei Arbeiterinnen und Drohnen und 19 Fraktionen bei der Königin.

Wir bezeichneten die Fraktionen mit den Buchstaben A-Y, ihr Auftreten wird auf Tabelle I und Abbildung 1 dargestellt. 
Tabelle I. Durch Elektrophorese in Polyacrylamidgel nachgewiesene wasserlösliche Proteine bei Apis mellifera $\mathrm{L}$.

+ Auftreten; - Fehlen.

\begin{tabular}{|c|c|c|c|c|c|c|c|}
\hline $\begin{array}{l}\text { Stadien } \\
\backslash \\
\text { Fraktionen }\end{array}$ & Eier & Rundmaden & Streckmaden & Puppen & Arbeiterinnen & Königin & Drohnen \\
\hline A & - & - & + & - & + & + & + \\
\hline B & + & + & + & + & + & + & + \\
\hline C & - & - & + & - & + & + & + \\
\hline D & + & + & + & + & + & + & + \\
\hline E & - & + & - & - & - & - & - \\
\hline $\mathrm{F}$ & - & + & + & + & - & - & - \\
\hline G & - & - & - & - & + & + & + \\
\hline $\mathrm{H}$ & - & + & + & + & + & + & + \\
\hline I & - & - & - & + & + & + & + \\
\hline $\mathrm{J}$ & + & + & + & + & + & + & + \\
\hline K & - & + & + & - & + & + & + \\
\hline L & + & + & + & + & - & - & - \\
\hline M & - & - & - & - & - & + & + \\
\hline $\mathrm{N}$ & - & - & - & - & + & + & - \\
\hline $\mathrm{O}$ & - & + & + & - & + & + & + \\
\hline $\mathrm{P}$ & + & + & + & + & + & + & + \\
\hline $\mathrm{R}$ & + & + & + & + & + & + & + \\
\hline S & + & + & + & + & + & + & + \\
\hline $\mathrm{T}$ & - & + & + & - & + & + & + \\
\hline $\mathrm{U}$ & - & + & - & + & + & + & + \\
\hline V & + & + & + & + & + & + & + \\
\hline W & + & + & + & + & - & - & - \\
\hline X & - & - & + & - & + & + & + \\
\hline Y & + & + & + & + & - & - & - \\
\hline
\end{tabular}

\subsection{Stärkegel}

Die Auftrennung im Stärkegel ergab bei den einzelnen Entwicklungsstadien der Bienen insgesamt 20 Banden, die wir mit den Buchstaben SA-SU kennzeichneten (Tab. II, Abb. 2). Bei den Eiern erhielten wir keine sichtbaren Bande. Bei Imagines beider Geschlechter fanden wir unterschiedliche Anzahlen von Fraktionen: bei Arbeiterinnen 9, bei der Königin 12 und bei Drohnen 11. Die für Königinnen charakteristischen Fraktionen SA und SB liegen im Anodenbereich, die für Drohnen typischen Banden ST und SU lagen im Kathodenbereich (Tab. II, Abb. 2).

\subsection{Isoelektrische Fokussierung in ultradünnem Polyacrylamidgel}

Die isoelektrische Fokussierung in ultradünnem Polyacrylamidgel führte zu einer größeren Anzahl von Banden. Bei Eiern konnten 31 (17 Anoden- und 14 Kathodenfraktionen), bei Rundmaden 50 (33 Anoden- und 17 Kathodenfraktionen), bei Streckmaden 50 (33 Anoden- und 17 Kathodenfraktionen), bei Puppen 48 (33 Anoden- und 15 Kathodenfraktionen), bei Arbeiterinnen 52 (36 Anoden- und 15 Kathodenfraktionen) und bei Drohnen 48 (31 Anoden- und 17 Kathodenfraktionen) nachgewiesen werden (Abb. 3). 


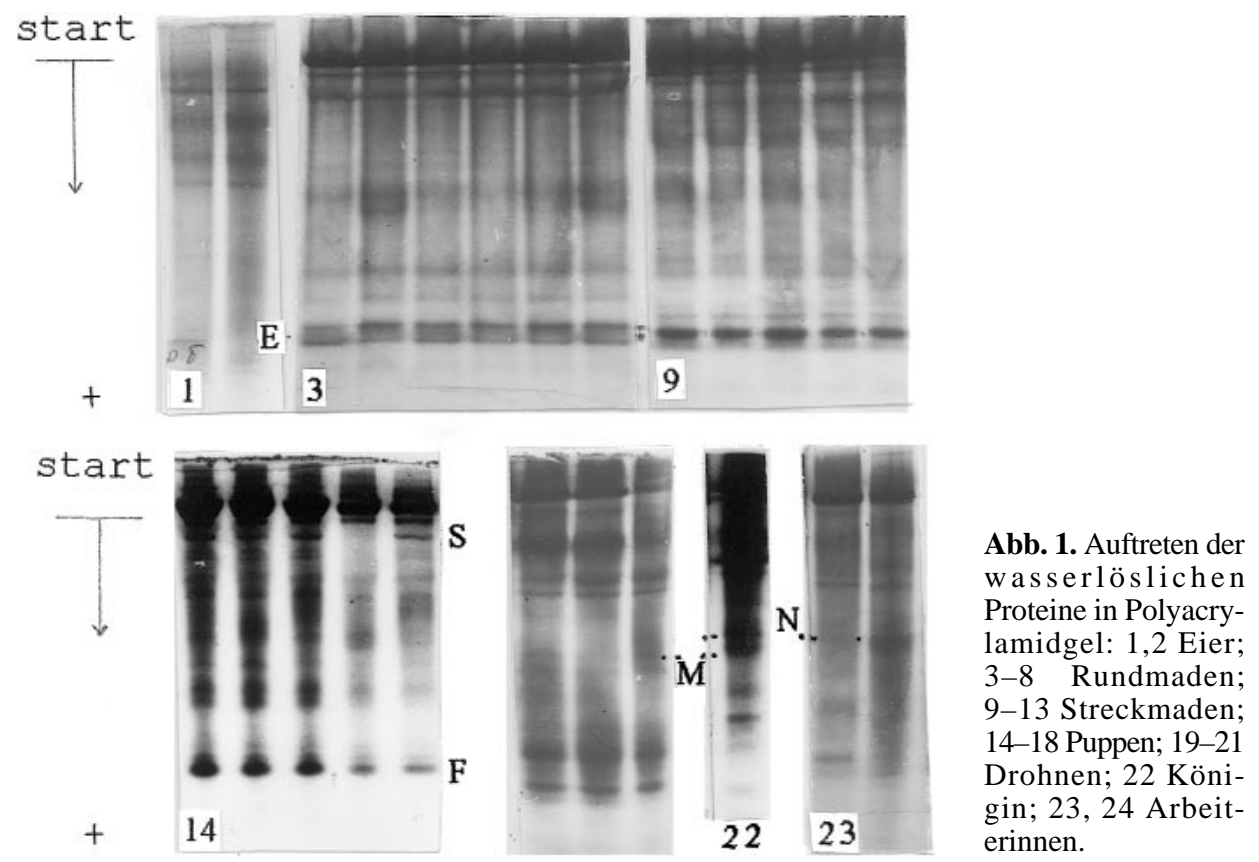

Tabelle II. Durch Elektrophorese in Stärkegel nachgewiesene wasserlösliche Proteine bei Apis mellifera $\mathrm{L}$.

+ Auftreten; - Fehlen.

\begin{tabular}{|c|c|c|c|c|c|c|}
\hline $\begin{array}{l}\text { Stadien } \\
1 \\
\text { Fraktionen }\end{array}$ & Rundmaden & Streckmaden & Puppen & Arbeiterinnen & Königin & Drohnen \\
\hline SA & - & - & - & - & + & - \\
\hline SB & - & - & _- & - & + & - \\
\hline $\mathrm{SC}$ & - & + & + & + & + & + \\
\hline SD & + & + & + & + & + & + \\
\hline SE & + & + & + & - & + & + \\
\hline SF & - & - & - & + & - & - \\
\hline SG & - & - & - & - & + & + \\
\hline SH & + & + & + & - & - & - \\
\hline SI & - & + & + & + & + & - \\
\hline SJ & + & + & + & + & + & + \\
\hline SK & - & + & + & - & - & + \\
\hline SL & + & + & + & + & + & + \\
\hline SM & + & - & - & + & + & - \\
\hline SN & + & - & _ & + & + & + \\
\hline SO & - & + & + & - & - & - \\
\hline SP & + & - & - & - & - & - \\
\hline SQ & - & + & + & - & - & - \\
\hline SR & - & + & - & + & + & + \\
\hline ST & - & - & _- & - & - & + \\
\hline SU & - & - & - & - & - & + \\
\hline
\end{tabular}




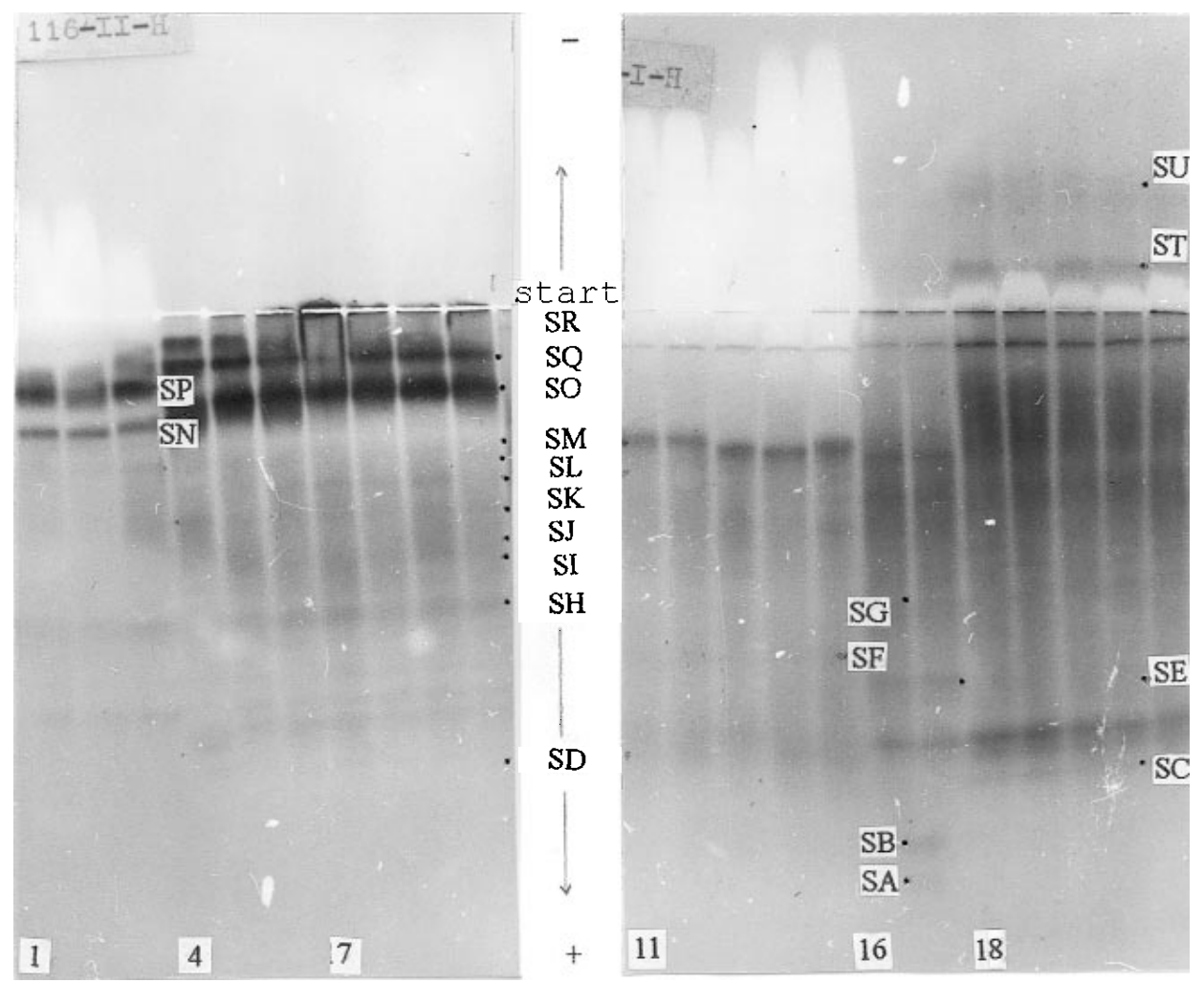

Abb. 2. Auftreten der wasserlöslichen Proteine in Stärkegel. 1-3 Rundmaden; 4-6 Streckmaden; 7-10 Puppen; 11-15 Arbeiterinnen; 16, 17 Königin; 18-22 Drohnen.

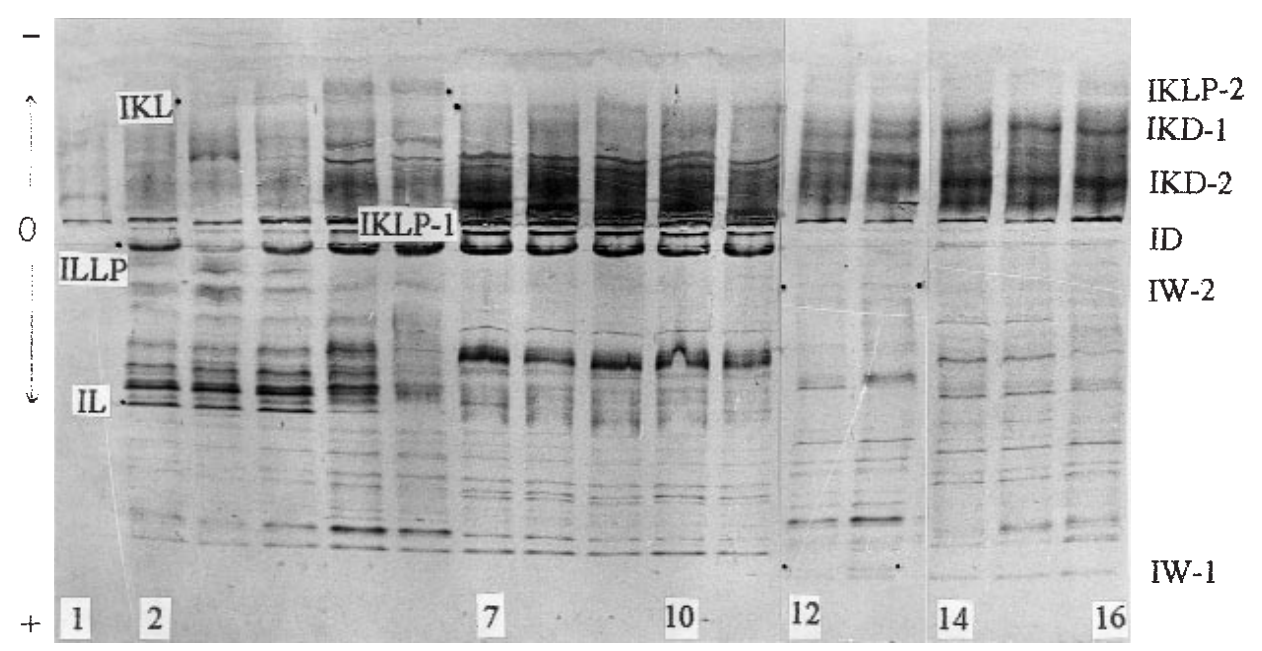

Abb. 3. Isoelektrische Fokussierung in ultradünnem Polyacrylamidgel: 1 Eier; 2-6 Rundmaden; 7-9 Streckmaden; 10-11 Puppen; 12-13 Arbeiterinnen; 14-16 Drohnen. 
Mit IL bezeichnen wir die Bande in der Anodenzone die für Rundmaden typisch sind, mit ILP die stark ausgeprägte Bande bei Streckmaden und Puppen. ILLP Bande sind im Larven- und Puppenstadium ausgeprägt. In der Kathodenzone erscheinen Bande, die wir als IKL, IKLP-1 und IKLP-2 bei Rundmaden, Streckmaden und Puppen bezeichneten.

Bei adulten Arbeiterinnen und Drohnen waren 3 Fraktionen aus der Anodenzone wichtig, die mit IW-1, IW-2 und ID gekennzeichnet wurden, und zwei Fraktionen der Kathodenzone, bezeichnet als IKD-1 und IKD-2. Die Proteinbanden IW-1 und IW-2 kommen nur bei Arbeiterinnen, ID, IKD-1 und IKD-2 nur bei Drohnen vor.

\section{DISKUSSION}

Die Elektrophorese in Polyacrylamidgel ergab eine Variation in der Zahl der Eiweißfraktionen von 10 bis 19 in den einzelnen Entwicklungsstadien, wobei ihre Anzahl im Spektrum der Streckmaden und der Imagines am größten ist.

Die Auswirkung der Wachstums- und Morphogenesestadien auf die Zahl der Fraktionen soll diskutiert werden. Die für Rundmaden charakteristischen Prozesse sind hauptsächlich mit ihrem Wachstum verbunden, während bei Streckmaden Metamorphoseprozesse in Verbindung mit der Histogenese bei den Puppen vorherrschen.

Die Ergebnisse zeigen, dass eine Veränderung im Vorkommen der Proteine während der Ontogenese der Bienen besteht. Allerdings zeigten sich nur bei zwei Trennungsmethoden bei den Streckmaden die meisten Banden (PAGE: 17 Fraktionen bei Rundmaden, 19 bei Streckmaden und 14 bei Puppen; Stärkegel: 8 Fraktionen bei Rundmaden, 11 bei Streckmaden und 10 bei Puppen). Nach Shao Wen Li et al. [27] tritt im Eiweißspektrum der Larven ein "Larvenprotein" auf, vermutlich Arylphorin [24], das bei den Imagines verschwindet. Unsere
Untersuchungen weisen ebenfalls in diese Richtung.

Im PAGE Spektrum von adulten Arbeiterinnen und Drohnen ist die Zahl der nachweisbaren Fraktionen 18, bei Königinnen 19, wobei nur bei den weiblichen (Arbeiterinnen und Königin) die Fraktion N auftritt, und bei Drohnen und Königin die Fraktion M (Tab. I, Abb. 1).

Shao Wen Li et al. [27] stellen durch PAGE das Auftreten von 11 Fraktionen bei Arbeiterinnen fest. Fraktion Nr. 5 ist nach den Autoren bei den untersuchten weiblichen Individuen Vitellogenin. Sabatini \& Fluri [25] fanden einen höheren Gehalt an Vitellogenin bei Königinnen im Vergleich zu Arbeiterinnen. Beim Vergleich mit unseren Ergebnisse halten wir eine Ähnlichkeit oder Übereinstimmung zwischen der nur bei weiblichen Individuen auftretenden NFraktion mit der Vitellogeninfraktion für möglich.

Das Spektrum der wasserlöslichen Proteine in der Stärkeplatte zeigt Unterschiede zwischen adulten Arbeiterinnen, Königinnen und Drohnen (Tab. II, Abb. 2). Für alle Imagines ist das Auftreten der Fraktionen SC, SD, SJ, SL, SN und SR charakteristisch, wobei zwei von ihnen, SC und SN, bei weiblichen Individuen (Königinnen und Arbeiterinnen) schwach und bei männlichen stark vertreten sind.

Die SF-Fraktion tritt nur bei Arbeiterinnen auf und SM bei allen weiblichen Individuen (Königinnen und Arbeiterinnen), nicht bei adulten Drohnen. Da im PAGE Spektrum auch eine typisch weibliche Fraktion N vorkommt, wird unsere Auffassung bestärkt, dass es für weibliche Individuen spezifische wasserlosliche Proteine gibt.

Bei Königinnen und Drohnen sind zwei Fraktionen ausgeprägt SE- und SG. SE fehlt nur bei Arbeiterinnen, SG außerdem auch bei Larven und Puppen. Bei der Trennung im Stärkegel ist die Fraktion SE bei allen Entwicklungsstadien vorhanden mit Ausnahme der adulten Arbeiterinnen. Die 
Fraktion SG kommt nur bei Königinnen und Drohnen vor. Auch bei PAGE erhielten wir eine nur bei Drohnen und Königinnen vorkommende Fraktion M. Das legt die Vermutung nahe, dass es sich ein für Geschlechtstiere typische Eiweißfraktion handeln könnte.

Es fanden sich 2 jeweils für Geschlechtstiere typische Fraktionen auf Stärkegelplatten: nur bei Proben von Königinnen traten die beiden Fraktionen SA und SB im Anodengebiet auf, die Fraktionen ST und SU dagegen traten nur bei Drohnen in der Kathodenzone auf (Tab. II, Abb. 2). Diese Produkte fehlen im Larven-, Vorpuppenund Puppenstadium der Ontogenese der Bienen, und ebenso bei allen weiblichen Imagines.

Nach der Feinfraktionierung mit isoelektrische Fokussierung in ultradünnem Servalytgel erscheint eine große Anzahl von Banden, von denen einige spezifisch für ein bestimmtes Stadium der Ontogenese, andere charakteristisch für alle Onotgenesestadien von Apis mellifera sind (Abb. 3). So sind die Bande IL für Rundmaden spezifisch, ILP für Streckmaden und Puppen, ILLP kommt im Larven- und Puppenstadium vor, IKL, IKLP-1 und IKLP-2 bei Rundmaden, Streckmaden und Puppen.

Die Fraktionen IW-1 und IW-2 kommen nur bei Arbeiterinnen, ID, IKD-1 und IKD-2 nur bei Drohnen. Das weist nach unserer Meinung auf einen Geschlechtsdimorphismus bei Apis mellifera hin.

Auf der Basis unserer Ergebnisse können wir verallgemeinern, dass wir durch die verschiedenen Methoden der nativen Elektrophorese eine Dynamik des Auftretens der Proteine im Verlauf der Ontogenese sowie auch Unterschiede zwischen beiden Geschlechtern feststellen können.

Summary - Electrophoretic study of water-soluble proteins during the honeybee (Apis mellifera L.) ontogenesis.
Two thousand specimens of Apis mellifera L. from three domesticated populations in Bulgaria were investigated by means of polyacrylamide gel electrophoresis (PAGE), starch gel electrophoresis (SGE) and isoelectric focusing in ultra-thin poyacrylamide gel. Specimens of different developmental stages and sexes were compared: unsealed and sealed larvae, white-eyed and dark-eyed pupae, and imago forms (7-day worker bees, 10-day drones, and one-day queen). All samples were total extracts of individuals, except the egg samples which were made up of 20 eggs. PAGE was performed according to Maurer [18]. SGE was performed according to Smithies [28] as modified by Dobrovolov [3]. The soluble proteins were visualised with Commassie Brilliant Blue R250.

A total of 24 fractions of water-soluble proteins were found in the spectra of analyzed organs in PAGE: 10 in eggs, 17 in unsealed larvae, 18 in sealed larvae, 14 in pupae, 18 in workers and drones and 19 in queens (Tab. I, Fig. 1). In SGE 20 fractions were found: 8 in unsealed larvae, 11 in sealed larvae, 10 in pupae, 9 in workers, 11 in drones and 12 in queens (Tab. II, Fig. 2). With isoelectric focusing 31 fractions were found in eggs, 50 in unsealed and sealed larvae, 48 in pupae, 52 in workers and 48 in drones (Fig. 3).

After separation in starch gel we found 2 queen specific and 2 drone specific bands. With PAGE, more bands were obtained but only one sex specific band (M) could be found. The best separation with up to 52 fractions was obtained by isoelectric focusing. There was 1 band at the anode and 2 bands at the cathode which occurred only in drones. With both SGE and PAGE we found protein bands which occurred during the larval and pupal but not in the adult stages (SQ and SH - F, L, W and Y respectively) which may point to specific larval proteins.

Apis mellifera / ontogenesis / watersoluble proteins 
Résumé - Étude par électrophorèse des protéines hydrosolubles au cours de l'ontogenèse chez l'abeille Apis mellifera $\mathbf{L}$. Deux mille abeilles (Apis mellifera L.) de Bulgarie ont été étudiées par électrophorèse sur gel de polyacrylamide (EGPA), sur gel d'amidon (EGA) et par isoélectrofocalisation en gel ultrafin de polyacrylamide à divers stades de développement. Nous avons comparé des œufs, des larves de 5 et 7 jours, des nymphes de 3 jours aux yeux blancs et de 7 jours aux yeux noirs, des ouvrières de 7 jours, des mâles de 10 jours et des reines âgées d'un jour. Tous les échantillons étaient constitués d'extraits totaux d'individus, sauf les échantillons d'œufs qui comportaient 20 œufs. Un tampon de tris-phosphate à pH 6,7 a été utilisé comme milieu d'extraction pour l'EGPA et de l'eau distillée pour l'EGA. Les échantillons ont été extraits pendant $24 \mathrm{~h}$ à $4{ }^{\circ} \mathrm{C}$, puis centrifugés durant $45 \mathrm{~min}$ pour l'EGPA et $30 \mathrm{~min}$ pour l'EGA à 900 g. L'EPGA a été faite selon Maurer [18] et l'EGA selon Smithies [28] modifié par Dobrovolov [3]. Les protéines solubles ont été visualisées à l'aide du bleu de Coomassie R-250.

L'EPGA a fourni un total 24 bandes de protéines hydrosolubles : 10 chez les œufs, 17 chez les larves non operculées, $18 \mathrm{chez}$ les larves operculées, 14 chez les nymphes, $18 \mathrm{chez}$ les ouvrières et les mâles et $19 \mathrm{chez}$ les reines (Tab. I, Fig. 1). L'EGA a fourni 20 bandes : 8 chez les larves non operculées, $11 \mathrm{chez}$ les larves operculées, $10 \mathrm{chez}$ les nymphes, 9 chez les ouvrières, 11 chez les mâles et 12 chez les reines (Tab. II, Fig. 2). L'isoélectrofocalisation a fourni un nombre de bandes beaucoup plus élevé : $31 \mathrm{chez}$ les œufs, 50 chez les larves operculées et non operculées, 48 chez les nymphes, 52 chez les ouvrières et 48 chez les mâles (Fig. 3). Après séparation sur gel d'amidon on a trouvé deux bandes spécifiques des reines et deux bandes spécifiques des mâles. Avec l'EGPA on a obtenu un plus grand nombre de bandes mais une seule est spécifique du sexe (M). La meilleure séparation, qui a fourni jusqu'à 52 fractions, a été obtenue par l'isoélectrofocalisation. Il y avait une bande à l' anode et deux à la cathode qui n'étaient présentes que chez les mâles. Avec l'EGA comme l'EGPA nous avons trouvé des bandes protéiniques présentes seulement chez la larve et la nymphe mais absentes des stades adultes (SQ et SH ( $\mathrm{F}, \mathrm{L}, \mathrm{W}$ et $\mathrm{Y}$ respectivement) qui peuvent désigner des protéines spécifiques des larves.

\section{Apis mellifera / ontogenèse / protéine hydrosoluble}

\section{LITERATUR}

[1] Arbuthnot P.B., Cantill R.C., Herbum H.R., An electrophoretic characterization of African worker honey bee hemolymph proteins during development, Comp. Biochem. Physiol. B 74 (1983) 467-471.

[2] Danty E., Arnold G., Burmester T., Huet J.C., Huet D., Pernollet J.C., Masson C., Identification and developmental profiles of hexamerins in antenna and hemolymph of the honeybee, Apis mellifera, Insect Biochem. Mol. Biol. 28 (1998) 387-397.

[3] Dobrovolov I.S., Mikroelektroforeza varchu skorbelen gel, Izvestija Niors-Varna 12 (1973) 157-162.

[4] Domackaja T.F., Grobov O.F., Gemolimfa pcel pri varoatoze, Pchelovodstvo 7 (1980) 8-12.

[5] Engels W., Fahrenhorst H., Age-dependent and caste-dependent changes in hemolymph protein patterns of Apis mellifera, Wilhelm Roux Arch. Entwickl. Mech. Org. 174 (1974) 285-296.

[6] Fluri P., Wille H., Gerig L., Lüscher M., Juvenile hormone, vitellogenin and hemocyte compositionin winter worker honeybees (Apis mellifera), Experientia 33 (1977) 1240-1241.

[7] Glinski Z., Jarosz J., Alterations in hemolymph proteins of drone honey bee larvae parasitized by Varroa jacobsoni, Apidologie 15 (1984) 329-338.

[8] Glinski Z., Jarosz J., Polyacrylamide gel electrophoresis in observations on insect haemolymph, Med. Wet. 3 (1986) 159-164 (in Polish).

[9] Glinski Z., Jarosz J., Werniski A., An immunoelectrophoretical analysis of soluble bload proteins of coiled and upright larvae of the worker honey bee, Apis mellifera, Apidologie 16 (1985) 247-265.

[10] Halberstadt K., Elektrophoretische Untersuchungen zur Sekretiontätigkeit der Hypopharynxdrüse der Honigbiene (Apis mellifera L.) Insectes Soc. 27 (1980) 61-77. 
[11] Huang Z.Y., Otis G.W., Teal P.E., A Nature of brood signal activating the protein syntesis of hypopharynge gland in honey bees, Apis mellifera (Apidae, Hymenoptera), Apidologie 20 (1989) 455-464.

[12] Ivanova E., Izmencivost pri Apis mellifera ontogeneticni i populacionno-geneticni aspekti, Dissert. Univ. Plovdiv, 1996.

[13] Kolev D.A., Shabanov M., Changes in the haemolymph proteins of drone larvae and pupae parasitized by Varroa jacobsoni Oud. 1904 Acta Microbiol. Bulg. 24 (1989) 41-44 (in Bulgarian, English summary).

[14] Krieg P., Marek M., Protein and esterase changes in the hemolymph of the queens of honey bee, Apis mellifera L., Comp. Biochem. Physiol. B-Biochem. \& Mol. Biol. 75 (1983) 513-517.

[15] Kubo T., Sasaki M., Nakamura J., Sasagawa H., Ohashi K., Takeushi H., Natori S., Change in the expression of hypopharyngeal gland proteins of the worker honeybees (Apis mellifera L.) with age and /or role, J. Biochem. 119 (1996) 291-295.

[16] Lin H.R., Winston M.L., Haunerland N.H., Slessor K.N., Influence of age and population size on ovarian development, and of trophallaxis on ovarian development and vitellogenin titres of queenless worker honey bee (Hymenoptera: Apidae), Can. Entomol. 131 (1999) 695-706.

[17] Lowry O., Rosebrough N.J., Farr A.L., Randall R.J., Protein measurement with the Folin pheno reagent, J. Biol. Chem. 193 (1951) 265-275.

[18] Maurer G., Disk-elektroforez, Mir, Moskva, 1971.

[19] Michelette E., Engels W., Concentration of hemolymph proteins duringpostembryonic worker development of Africanized honey bees in Brazil and Carniolans in Europe, Apidologie 26 (1995) 101-108.

[20] Ohashi K., Sawata M., Takeuchi H., Natori S., Kubo T., Molecular cloning of cDNA and analysis of expression of the gene far alpha-glucosidase from the hypopharyngeal gland of the honeybee Apis mellifera L., Biochem. Biophys. Res. Commun. 221 (1996) 380-385.

[21] Ohashi K., Natori S., Kubo T., Change in the mode of gene expression of the hypopharyngeal gland cells with an age-dependent role change of the worker honeybee Apis mellifera L., Eur. J. Biochem. 249 (1997) 797-802.

[22] Popov P., Ivanova E., Electrophoretic studies on the total soluble protein in imago forms of Apis mellifera L., Animalia 30 (1994) 53-56.

[23] Raolison C., Sutter B., An intestinal insulin-like molecule in Apis mellifera., Comp. Biochem. Physiol. A 69 (1981) 79-83.

[24] Ryan R.O., Schmidt J.O., Law J.H., Arylphorin from the haemolymph of the larval honeybee, Apis mellifera, Insect Biochem. 14 (1984) 515-520.

[25] Sabatini A., Fluri P., Blood juvenile hormone protein and vitellogenin titres in laying and nonlaying queen honey bee, J. Apic. Res. 20 (1981) 221-225.

[26] Schatton-Gadelmayer K., Engels W., Haemolymph-Proteine und Körpergewicht frischgeschlüpfter Bienen-Arbeiterinnen nach unterschiedlich starker Parasitierung durch Brutmilben, Entomol. Genet. 14 (1988) 93-101.

[27] Shao-Wen Li, Jang Qwen Mei, Meng Jo-Pin, Chang I., Studies on the haemolymph protein pattern of two species of honey bees Apis mellifera, Acta Entomol. Sin. 25 (1982) 185-190 (in Chinese).

[28] Smithies O., Zone electrophoresis in starch gels, Biochem. J. 61 (1955) 629-641.

[29] Trenczek T., Zillikens A., Engels W., developmental patterns of vitellogenin hemolymph titer and rate of syntesisin adult drone honey bees (Apis mellifera), J. Insect Physiol. 35 (1989) 475-481.

[30] Wheeler D.E., Kawooya J.K., Purification and characterization of honey bee Vitellogenin, Arch. Insect Biochem. Physiol. 14 (1990) 253-267.

[31] Zillikens A., Age and juvenilehormone-dependent vitellogenin syntesis in imaginal honey bee drones, Apidologie 16 (1985) 237-238.

To access this journal online: www.edpsciences.org 